\title{
Information and measuring system for operational monitoring of soil moisture and temperature
}

\author{
Yuri Blokhin", Svetlana Blokhina, Anna Dvirnik, Andrei Belov, and Andrei Makushin \\ Agrophysical Research Institute, 195220, St. Petersburg, Grazhdansky Prosp.14, Russia
}

\begin{abstract}
The structure of the wireless sensor network (WSN) for operational monitoring of soil moisture and temperature on the agricultural field with high accuracy is considered in the article. The evaluation of wireless sensor networks' technical capabilities for agricultural applications is given; the advantages and disadvantages of their use in the field conditions are noted, taking into account the influence of different factors on the quality of communication. To solve the problems of radio communication in the soil and deploying a sensor device completely below the ground surface, the prototype model of a sensor node with a retractable antenna has been developed. Sensor nodes were communicated via the ZigBee protocol and the integrated spatial attribute information was transmitted into the database.
\end{abstract}

\section{Introduction}

Modern remote sensing technologies, sensor technologies and IoT technologies will play a key role in various applications of the agricultural sector, but today there are real possibilities for remote monitoring of agricultural fields. Information and measuring systems (IMS), including wireless sensor networks (WSN), facilitate the monitoring of soil conditions with high accuracy and can efficiently detect early stages of adverse state at fields. For this reason, modern agriculture requires the use of "smart" sensors and mobile systems from planting to harvesting. Timely data acquisition using IMS makes the whole process not only "smart", but also cost-effective due to accurate monitoring capabilities [1, 2].

A wireless sensor network (WSN) is a sensing system consisting of a group of spatiallydistributed sensors for automatic and wireless monitoring of a variety of soil conditions over an area of several square meters to several kilometers, which has the property of selforganization of many sensor nodes and actuators, interconnected via a radio channel.

In recent years, WSN have been rapidly developed and reached a new technological level due to cost reduction, sensor device compactness and advances in radio frequency technology and digital communication system. WSN are a promising tool for environmental monitoring, irrigation, animal husbandry, greenhouse complexes and other

\footnotetext{
*Corresponding author: blohin3k4@gmail.com
} 
agricultural industries [3, 4, 5], and have the following advantages: autonomy, faulttolerance, scalability, various network topologies, multithreaded communication, tolerance for communication failures in severe environmental conditions, information security and reasonable price, available to a wide range of end users. However, the WSN has a number of shortcomings, such as limited battery charge, low performance and relatively low flash data memory of sensor node controllers.

\section{Materials and methods}

\subsection{Application of WSN for operational monitoring of soil moisture and temperature}

WSN technologies have attracted a large number of research efforts in soil moisture and temperature monitoring over the past few years $[3,4]$, due to high availability of standards: Bluetooth Low-Energy, Zigbee, Z-Wave, 6LoWPAN, LoRa. Relatively low equipment costs provide the installation of a large number of sensor nodes to investigate the spatial heterogeneity of agrophysical characteristics on the experimental fields. WSN can be integrated with the geographic information system.

The usefulness of WSN is clear for collecting, transmitting and processing data on soil temperature and moisture in real time from a certain quantity of sensor nodes to obtain information about soil conditions.

Structural scheme of WSN for operational monitoring of soil properties has been developed (Fig. 1). The WSN consists of the base station and the required number (from 1 to n) of sensor nodes installed across the fields using the "mesh" topology. Power conservation is a primary concern in the design of the sensor network. Conservation can be achieved by utilizing power-efficient hardware and communication protocols, so the ZigBee protocol was chosen for WSN radio communications. The strategy of the spatial location of stationary sensor nodes of WSN is based on heterogeneity zone delineation using remote sensing data and/or mobile complex for determination of agrophysical characteristics of arable soil layer in real time [5]. Each network has usually only a single base station which controls and keeps the network running and streams information from the sensor nodes to the internet server with a web interface on which the field measurement database is generated. The base station of the network consists of: a microcomputer, power supply unit $(220 \mathrm{~V})$, a $4 \mathrm{G}$ modem and a ZigBee-router. The sensor node consists of: a fivechannel borehole dielcometric soil moisture meter, a ZigBee-router, a battery and an external antenna. In general, electromagnetic water content sensors are well suited for monitoring soil moisture profiles at a depth of up to $1 \mathrm{~m}$ with high temporal resolution. Special Java-based software has been developed for the WSN microcomputer, and the AT commands and assembler language are used for the modem and sensor nodes microcontroller. Access to the database, remote control of WSN, and updating the software of the network nodes are carried out through a specially designed website. 


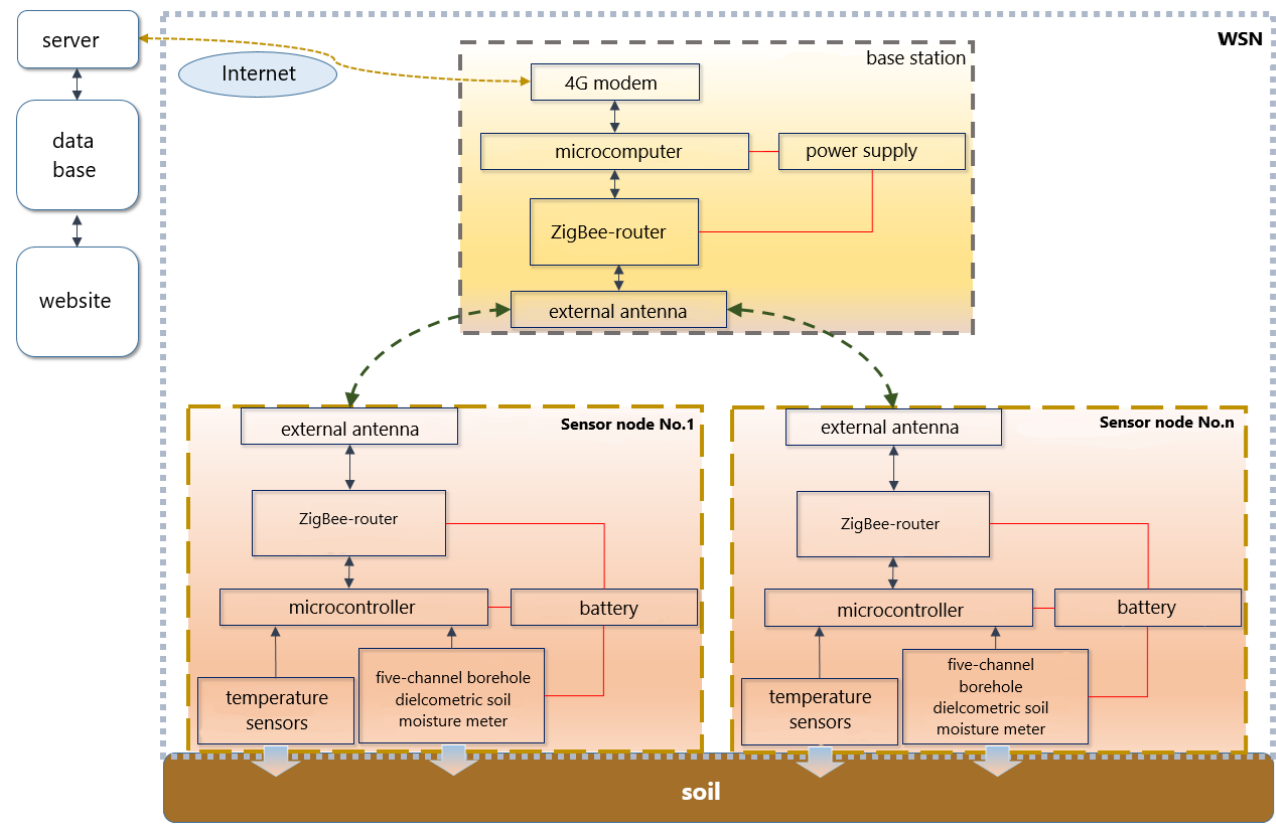

Fig. 1. Structural scheme of WSN for operational monitoring of soil properties

\subsection{WSN application challenges in agriculture}

WSN should operate in different field conditions, taking into account the topographic features of a particular area and weather conditions. Radio communication between the transmitters of WSN node at a real agricultural field is complicated by multi path spreading and fading effects, reflection from the soil surface and signal attenuation. When placing WSN on an agricultural field, it is important to determine the optimal location, the distance between sensor nodes and the height of the antenna, with due regard for plant biomass gain during the growing season. The increase of the leaf surface area leads to the attenuation and changes in the intensity of the receiving signal, especially when the sensor node antenna is deployed near the soil surface, in plant rows or across them [6].

Another factor affecting the communication quality of WSN nodes is climate conditions. The effects of moisture due to humidity, precipitation and wetting influence the communication links caused the attenuation, amplification or loss of signal. In addition, ambient temperature affects the sensor nodes performance. Low temperatures have a negative effect on the battery life of the nodes. Measurements made by sensor nodes can be erroneous if the battery voltage drops due to a decrease in temperature below a certain threshold.

The WSN should function in the fields with due regard for technological operations that are utilized by agricultural equipment such as tractors, that can cause damage to the devices.

The placement of aboveground WSN stationary sensor nodes is impossible during the perfoming of main soil treatment practices (ploughing, plowing, cultivation, harrowing) because of aboveground and underground sensor node parts, especially the antenna, are vulnerable to working elements of agricultural equipment such as plows.

The placement of WSN in the field after planting is complicated by technological operations of plant protection, fertilization and top dressing, which can threaten the 
integrity of the aboveground part of the sensor nodes. The spraying boom can pass at a height of several centimeters to a couple of meters from the ground surface at the WSN sensor node location, which may coincide with the height of the sensor node antenna. Usually, before conducting technological operations, the wireless devices deployed in the field may need to be removed or lowered to the ground to avoid damage. However, this causes inconvenience for users and interruption of data collection.

\subsection{Wireless underground sensor networks}

The need to conduct technological operations in the fields using agricultural equipment leads to the necessity of sensor nodes to be deployed below the ground surface as components of the wireless underground sensor network (WUSN) for operational monitoring of soil moisture and temperature. The sensor node contains all the necessary components for the self-contained operation in the field during the growing season, which is different from WSN, where some parts of the sensor nodes are deployed above the ground.

The underground environment is far from ideal place for placing electronic devices. Moisture, temperature, internal soil pressure, humans, animals, insects and earth moving equipment pose a physical threat to WUSN nodes. Microcontrollers, routers, power supplies source, and other node components must be placed in a protective enclosure and be resilient to negative factors.

Underground wireless communication is more challenging than wireless open-air communication.

WUSNs are an exciting research area because of the unique nature of the underground environment such as water content, particle sizes, density, temperature, moisture and soil heterogeneity directly effect on connection, data transmission and reception quality in the wireless network [7].

\section{Results and Discussion}

The scheme of the sensor node for deploying in the field below the ground (to avoid damage from agricultural equipment) as a component of the WUSN for operational monitoring of moisture and temperature in the soil profile with high temporal resolution (every 10 minutes) is presented on Fig.2. Sensor nodes and the base station communicate by means of the retractable antenna. The linear actuator creates motion of the external antenna from the borehole to a height of $1 \mathrm{~m}$ above ground with a force of $300 \mathrm{~N}$ and a speed of $48 \mathrm{~mm} / \mathrm{s}$. After successful data transmission, the actuator returns the antenna to the bore hole, tightly closes the outlet preventing soil and moisture from entering.

After exiting standby mode, the microcontroller senses the state of the five-channel moisture meter, converts the received data to moisture and temperature values, controls the actuator motor operation with the relay and transmits data packets to the base station using the ZigBee- router to the WUSN base station. 

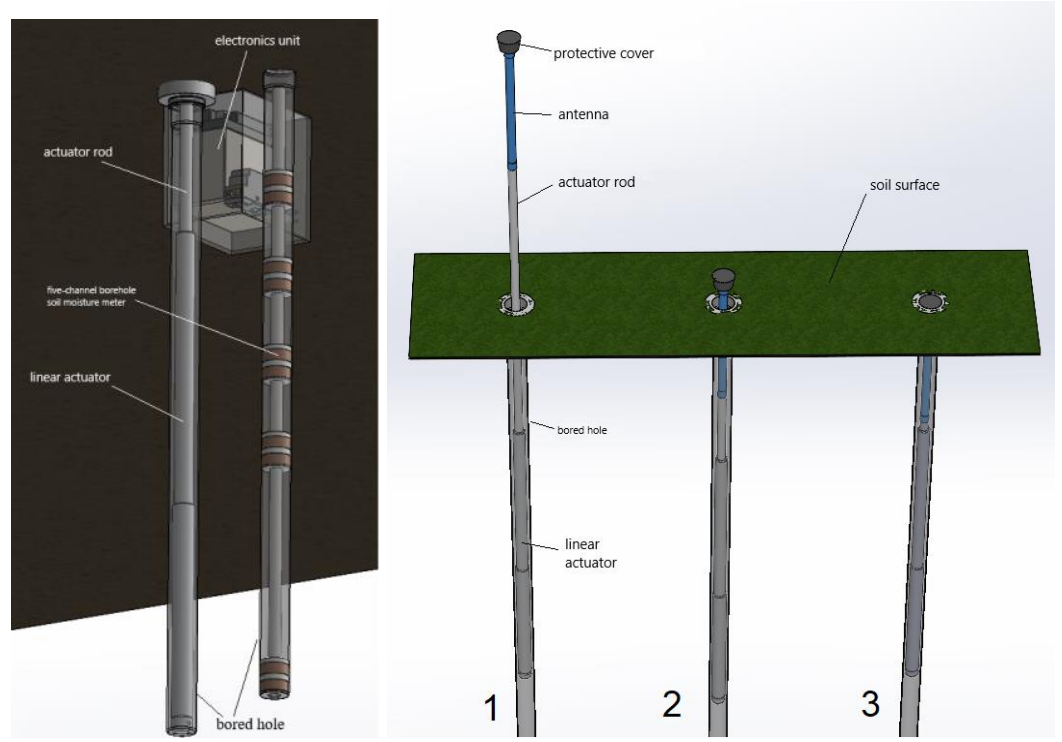

Fig. 2. The scheme of the sensor node and the antenna position out of the bored hole $(1-$ the antenna fully extended, 2 - the actuator returns the antenna back to the bored hole, 3 — the antenna is deployed completely below the surface of the ground, the bored hole is closed by a protective cover).

\section{Conclusions}

For monitoring of the spatial variability of soil moisture and temperature in the agricultural fields, the concept of WUSN in which sensor nodes are deployed completely below ground are considered. Integrated spatial-attribute data obtained using WUSN can be used for timely implementation of appropriate fertilization and plant protection management during the growing season.

\section{Acknowledgments}

This work was supported by the Federal budget under an agreement on granting from December 10, 2019 No. 05.607.21.0302, unique project identifier RFMEFI60719X0302.

\section{References}

1. M. Ayaz, M. Ammad-Uddin, Z. Sharif, A. Mansour, E. M. Aggoune, IEEE Access, 7, 129551-129583 (2019)

2. U. Shafi, R. Mumtaz, J. García-Nieto, S.A. Hassan, S.A.R. Zaidi, N. Iqbal, Sensors, 19, 3796 (2019)

3. R. Sui, J. Baggard, AEA, 31, 193-200 (2015)

4. T. Ojha, S. Misra, N.S. Raghuwanshi, CaEiA, 118, 66-84 (2015)

5. I.P. Ananev, V.S. Zubets, A.V. Belov, Yu.I. Blokhin, Proceedings 3rd GWPSS, 201209 (2013)

6. P. Andrade-Sanchez, F.J. Pierce, T.V. Elliot, ASABE, MN, 7, 10 (2007)

7. A. R. Silva, M. C. Vuran, ICC 2010, 1-6 (2010) 
8. J. Gutiérrez, J. F. Villa-Medina, A. Nieto-Garibay, M. Á. Porta-Gándara, IEEE Trans. IM, 63 (1), 166-176 (2014) 